Ann. Biol. anim. Bioch. Biophys., 1971, 11 (2), 213-224.

\title{
ÉTUDE COMPARATIVE DU FLUIDE UTÉRIN ET DE L'ALBUMEN DE L'CEUF IN UTERO CHEZ LA POULE
}

\author{
B. SAUVEUR et P. MONGIN \\ avec la collaboration technique de J. ROCARD \\ Station de Recherches avicoles, \\ Centre de Recherches de Tours, I. N.R. A., \\ 37 - Nouzilly
}

RÉSUMÉ

Afin d'étudier les transferts d'eau et d'ions effectués dans l'utérus de la poule, nous avons prélevé à cet endroit l'albumen d'œufs en formation et le fluide utérin pendant une période continue s'étendant de 6 à $\mathbf{2 4}$ heures après l'oviposition précédente. Ces prélèvements ont été effectués toutes les 2 heures, avec 6 animaux par stade. Pour les prélèvements qui auraient dû normalement être effectués pendant la péricde nocturne, nous avons utilisé des animaux placés en rythme nycthéméral inversé. Sur chaque échantillon d'albumen et de fluide utérin nous avons

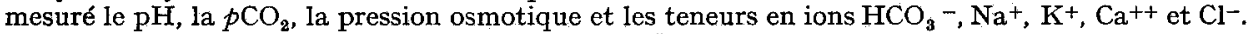

L'albumen et le fluide utérin sont le siège de flux ioniques qui peuvent s'inverser suivant le stade considéré : ainsi $\mathrm{Na}^{+}$est transféré vers l'albumen entre 6 et I 2 heures puis réabsorbé de I 2 à 22 heures après oviposition. Durant cette deuxième période un échange $\mathrm{Na}^{+} / \mathrm{K}^{+}$se produit entre l'albumen et le fluide utérin dans un rapport $\mathrm{I} / \mathrm{I}$ mais $\mathrm{il} \mathrm{n}^{\prime}$ est pas démontré qu'un transport couplé $\mathrm{Na}^{+} / \mathrm{K}^{+}$existe au niveau de la membrane utérine.

La $18 \mathrm{e}$ heure est caractérisée par une pression osmotique intra-luminale minima et par une acidose maxima due à une mobilisation d'ions $\mathrm{HCO}_{3}-$. Au début de la croissance pondérale de la coquille (Io heure) on observe une baisse de teneur en $\mathrm{Ca}^{++}$de l'albumen et un brusque enrichissement en $\mathrm{Ca}^{++}$du fluide utérin.

Ces résultats sont discutés en fonction des études déjà effectuées sur la provenance des ions $\mathrm{Ca}++$ et $\mathrm{CO}_{3}{ }^{--}$de la coquille ; ils confirment que des ions $\mathrm{HCO}_{3}$ - en provenance du $\mathrm{CO}_{2}$ métabolique doivent être sécrétés par les cellules utérines.

La nature des transferts qui interviennent dans l'hydratation de l'œuf in utero est également discutée ; il semble bien établi qu'à la suite de la phase de " plumping " (6-I 2 h), interviennent des échanges cationiques entre l'albumen, le fluide utérin et le sang. 


\section{INTRODUCTION}

Les transferts d'eau et d'électrolytes effectués à travers la paroi utérine des oiseaux sont d'une importance fondamentale pour la constitution de l'albumen et de la coquille de l'œuf. On sait en particulier que, chez la Poule, l'utérus fournit à l'albumen une quantité très importante d'eau et de potassium (DRAPER, I966; LEONARD, I968) ; les transferts de sodium, potassium, calcium et chlore effectués dans cet organe varient avec l'équilibre acido-basique de l'animal (SAUVEUR, I970 $b$ ) et modifient la structure physique de 1'albumen (SAUVEUR, I970 a).

Ainsi, la qualité de l'albumen et celle de la coquille dépendent pour partie ou en totalité des échanges ioniques accomplis in utero. Par ailleurs, la composition des sécrétions utérines peut influer sur le métabolisme des spermatozoïdes lors de leur remontée vers l'infundibulum, lieu de fécondation (EI, JACK et LAKE, I967).

Cependant le fluide présent dans l'utérus, autour de l'œuf, n'a été étudié qu'à quelques instants particuliers qui excluent toujours la période nocturne (BEADLE, CONRAD et SCOTT, I938; Ei, JACK et LAKE, I967 ; LEONARD, I968). C'est pourquoi il nous a semblé indispensable d'en entreprendre une étude continue qui s'étende sur toute la durée du transit utérin de l'œuf et soit complétée par une analyse de l'albumen. Le présent rapport donne les résultats de ces observations et quelques hypothèses qui en résultent sur le mécanisme de transfert des ions in utero.

\section{MATÉRIEL ET MÉTHODES}

Cette expérience a été conduite sur des poules pondeuses White Leghorn (H. N.) placées en cages individuelles, éclairées $16 \mathrm{~h} / \mathrm{j}$ et recevant un régime standard pour reproducteurs. Vingt animaux environ furent placés en rythme nycthéméral inversé afin de permettre l'étude des stades de formation de l'œuf qui interviennent le plus fréquemment pendant la période nocturne.

L'heure de ponte de chaque œuf étant enregistrée automatiquement, nous avons, après confirmation de la présence d'un cuf in utero par palpation à travers le cloaque, prélevé des ceufs en formation et du fluide utérin à intervalles de temps réguliers après oviposition de l'cuf précédent.

Ces prélèvements, 6 à chaque stade, cnt été effectués sous anesthésie générale de l'animal (par courant de $\mathrm{O}_{2}+\mathrm{N}_{2} \mathrm{O}+$ éther) par laparatomie et pression digitale sur la paroi utérine externe selon la méthode de EL JACK et LAKE (1967). L'œuf en formation est ainsi chassé après retournement du cloaque et le fluide utérin qui s'écoule est recueilli dans un tube conique.

Les mêmes méthodes d'analyse ont été appliquées à l'albumen et au fluide utérin : mesure du $\mathrm{pH}$ par microélectrode Radiometer $\left(\mathrm{E}_{5} \mathrm{O} 2 \mathrm{I}\right)$, de la $p \mathrm{CO}_{2}$ par la méthode d'Astrup (microtonomètre Radiometer) et calcul de la concentration des ions bicarbonates par l'équation d'Hasselbach-Henderson affectée des coefficients utilisés par SAUveuR (1969). La pression osmotique des échantillons a été déterminée par mesure de l'abaissement du point de congélation (osmomètre Fiske), les teneurs en $\mathrm{Na}^{+}, \mathrm{K}^{+}, \mathrm{Ca}^{++}$par spectrophotométrie de flamme (Eppendorf) et celles en $\mathrm{Cl}^{-}$par titration potentiométrique au nitrate d'argent (électrode Radiometer $\mathrm{P}_{4}$ Or).

\section{RÉSULTATS}

Les données numériques enregistrées ayant fait l'objet d'une publication partielle (Mongin et SAUvEUR, I970) nous donnerons principalement ici des figures illustrant les variations observées. 
Les teneurs en électrolytes de l'albumen sont rapportées, d'une part à la matière sèche du milieu, d'autre part à l'eau. Le premier mode d'expression s'appuie sur le fait que la matière sèche de l'albumen ne varie pratiquement plus dans l'utérus ; il représente donc une estimation des quantités totales d'ions présentes (SAUvEUR, I970 b).

I $^{\mathbf{0}}$ Le dépôt de la coquille (fig. I) s'effectue entre ro et 22 heures après ovulation de l'œuf, à la vitesse constante de $318 \mathrm{mg} / \mathrm{h}$. Entre ces deux instants le coefficient de corrélation entre le poids de coquille déposée et le temps est de 0,97 (calculé sur 27 couples de valeurs).

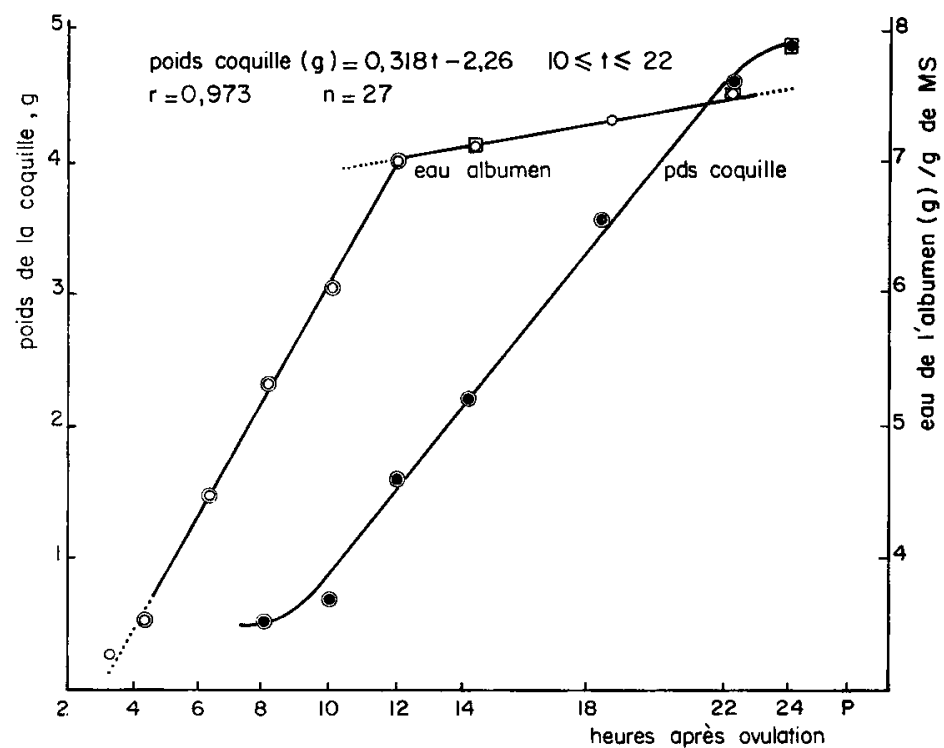

Fig. I. - Dépôt de la coquille (g) et apport d'eau à l'albumen (g/g matière sèche) en fonction du temps écoulé depuis l'oviposition précédente

(ㅇ)

四

- : valeur différente de celle du stade $(n-\mathrm{r})(\mathrm{P} \leqslant 0,05)$

Q : valeur différente de celle du stade $(n-2)(P \leqslant 0,05)$

$2^{\circ}$ L'enrichissement de 1'albumen en eau (fig. I) commence dès l'entrée de 1'œuf dans l'utérus et se poursuit jusqu'à la $I 2^{\mathrm{e}}$ heure après ovulation (soit 2 heures après le début de la calcification de la coquille) au taux de $45^{8} \mathrm{mg}$ eau/h/g de matière sèche ; pendant cette période le coefficient de corrélation entre la teneur en eau de 1'albumen et le temps est de 0,92 . Entre I2 et 22 heures, 1'hydratation se poursuit beaucoup plus lentement à travers la coquille : l'apport d'eau, linéaire en fonction du temps, n'est plus alors que de $38 \mathrm{mg} / \mathrm{h} / \mathrm{g}$ de matière sèche.

$3^{\text {o }}$ La teneur en sodium de l'albumen évolue de façon diphasique (î́g. 2). Entre la $6^{\mathrm{e}}$ et la $\mathrm{I}^{\mathrm{e}}$ heure après ovulation, il existe un flux net de sodium dirigé vers 1'albumen égal à $34 \mu$ éq/h/g de matière sèche ; ce transfert est donc simultané à 1'hydratation rapide décrite ci-dessus et correspond au phénomène couramment appelé " plumping " de 1'albumen. Entre 12 et 22 heures il se produit un flux net de sodium en sens opposé, dirigé de l'albumen vers les liquides extracellulaires, égal à I4 $\mu$ éq/h/g de matière sèche. L'abaissement constant de concentration du sodium dans l'albumen 


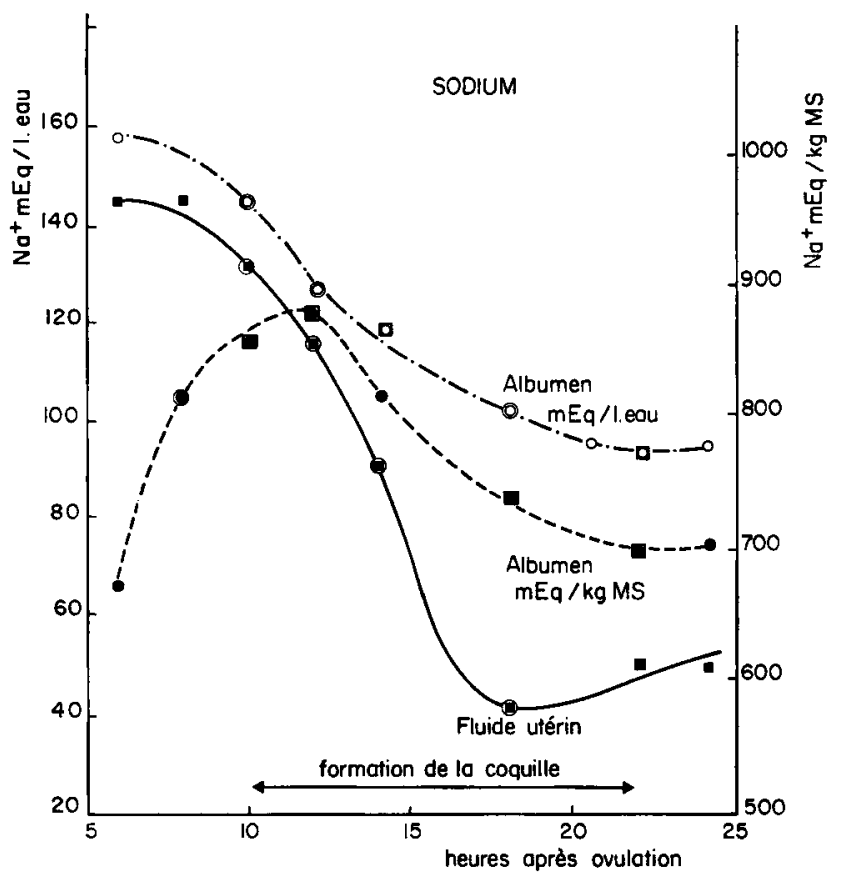

FIG. 2. - Teneur en sodium de l'albumen et du fuide utérin en fonction du temps écoulé depuis l'oviposition précédente - - - : en méq $/ \mathrm{kg}$ matière sèche de l'albumen $\mathrm{o}-\mathrm{o}$ : en méq/l d'eau de l'albumen $u$

- - en méq/l de fluide utérin

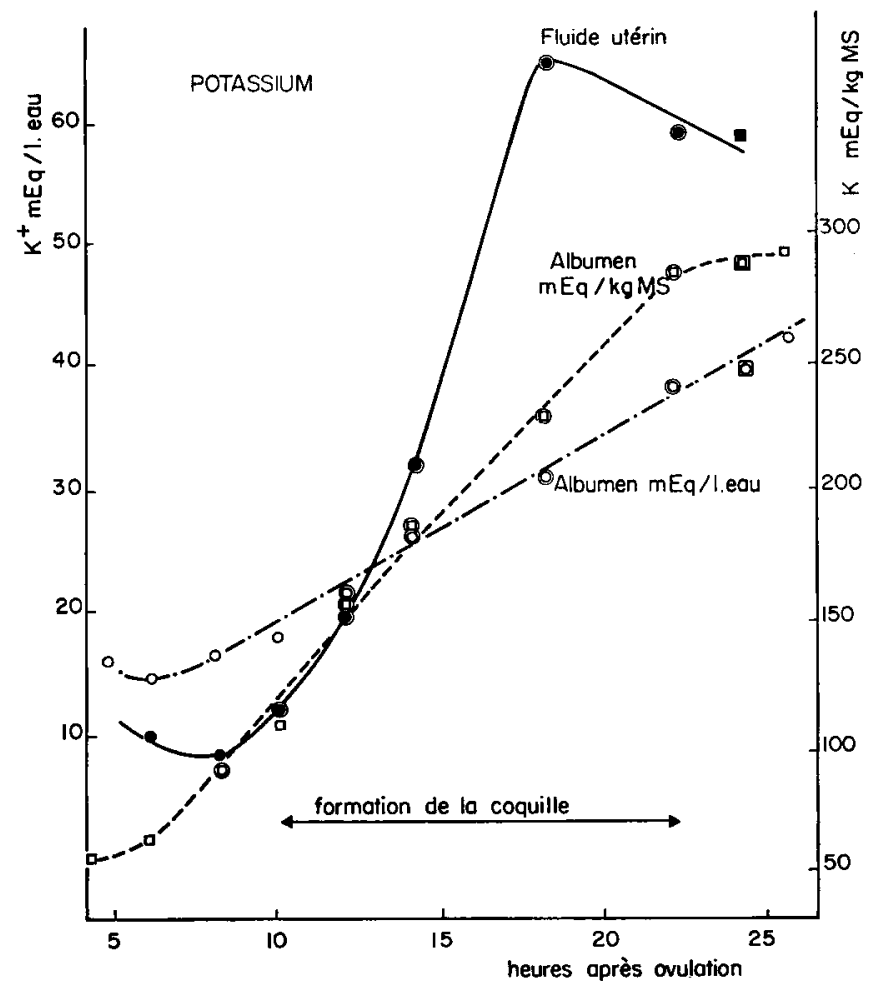

FIG. 3. - Teneur en potassium de l'albumen et du fuide utérin en fonction du temps écoulé depuis l'oviposition précédente Légende : voir fig. I et 2 


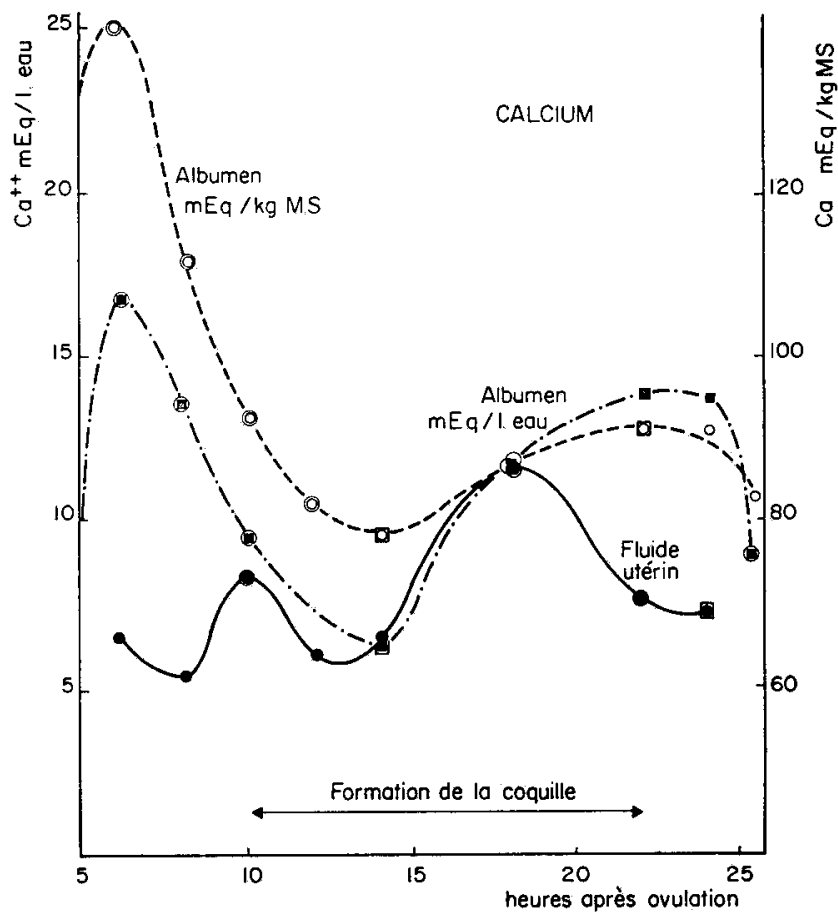

FIG. 4. - Teneur en calcium de l'albumen et au fiuide utérin en fonction du temps êcoulé depuis l'oviposition précédente Légende : voir fig. I et 2

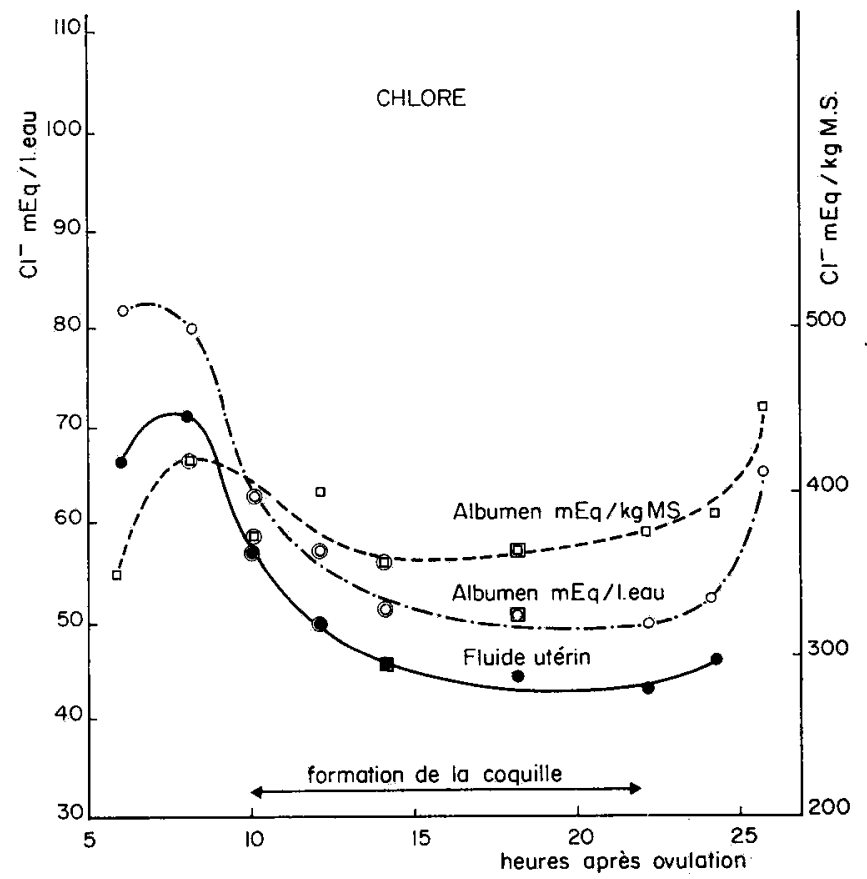

FIG. 5. - Teneur en chlore de l'albumen et du fuide utérin en fonction du temps écoulé depuis l'oviposition précédente Légende : voir fig. I et 2 
apparaît donc dû, jusqu'à I2 heures, à un flux hypoosmotique puis à un faible apport d'eau accompagné de la réabsorption de sodium évoquée ci-dessus.

$4^{\circ} \mathrm{Il}$ se produit un flux constant de potassium vers l'albumen ( $14 \mu$ éq/h/g de matière sèche) depuis l'entrée de l'œuf dans l'utérus jusqu'à la $22^{\mathrm{e}}$ heure (fig. 3 ). Pendant environ to heures, l'albumen s'enrichit donc en potassium à travers la coquille plus ou moins calcifiée. Remarquons de plus que, entre 12 et 22 heures, ce flux d'ion $\mathrm{K}^{+}$est, en valeur absolue, exactement identique à celui de $\mathrm{Na}^{+}$mais dirigé en sens opposé.

$5^{\circ}$ L'apport de calcium à l'albumen effectué dans l'isthme se termine 6 heures après ovulation (fig. 4) et est suivi d'une diminution du contenu calcique de ce milieu jusqu'à la $14^{\mathrm{e}}$ heure. Un nouvel apport est effectué in utero entre la $14^{\mathrm{e}}$ et la $22^{\mathrm{e}}$ heure. La concentration du calcium dans l'albumen suit les transferts nets.

$6^{\circ}$ Les flux d'ions $\mathrm{Cl}-$ (fig. 5) varient de façon identique à ceux du sodium, avec une amplitude et une signification moindres. L'albumen s'enrichit en chlore durant le début du "plumping » $(6-8 \mathrm{~h})$ puis son contenu diminue jusqu'à la $\mathbf{I} 8 \mathrm{e}$ heure. L'augmentation apparente de la teneur en chlore du blanc durant les dernières heures passées dans 1'utérus n'est pas significative.

\section{TABLEAU I}

Équilibre acido-basique de l'albumen et du fluide utérin au cours du séjour de l'œuf in utero

\begin{tabular}{|c|c|c|c|c|c|c|c|c|c|c|}
\hline \multicolumn{2}{|c|}{$\begin{array}{l}\text { Heures après } \\
\text { oviposition } \\
\text { précédente }\end{array}$} & 6 & 8 & 10 & 12 & $1 / 4$ & 18 & 22 & 24 & $\mathrm{~F}$ \\
\hline $\mathrm{pH}$ & $\begin{array}{c}\text { Albumen } \\
\text { f.u. }\end{array}$ & $\begin{array}{l}7,39 \\
7,64\end{array}$ & $\begin{array}{l}7,45 \\
7,61\end{array}$ & $\begin{array}{l}7,40 \\
7,45 a\end{array}$ & $\begin{array}{c}7,37 \\
7,52\end{array}$ & $\begin{array}{l}7,36 \\
7,34\end{array}$ & $\begin{array}{l}7,18^{a} \\
7,11^{a}\end{array}$ & $\begin{array}{l}7,12^{b} \\
7,13^{b}\end{array}$ & $\begin{array}{l}7,38^{a} \\
7,48^{a}\end{array}$ & $\begin{array}{l}10,9 * * * \\
14 * * *\end{array}$ \\
\hline $\begin{array}{c}p \mathrm{CO}_{2} \\
(\mathrm{mmHg})\end{array}$ & $\begin{array}{c}\text { Albumen } \\
\text { f.u. }\end{array}$ & 82 & $\begin{array}{l}103 \\
177,5\end{array}$ & $\begin{array}{l}108 \\
238\end{array}$ & $\begin{array}{r}105 \\
93\end{array}$ & $\begin{array}{l}110 \\
172\end{array}$ & $\begin{array}{r}94 \\
141\end{array}$ & $\begin{array}{l}114 \\
174\end{array}$ & $\begin{array}{l}111 \\
119\end{array}$ & $\begin{array}{l}1,6 \mathrm{NS} \\
2,0 \mathrm{NS}\end{array}$ \\
\hline $\begin{array}{l}\mathrm{HCO}_{3}- \\
(\mathrm{méq} . / 1)\end{array}$ & $\begin{array}{c}\text { Albumen } \\
\text { f.u. }\end{array}$ & 51,3 & $\begin{array}{l}41 \\
128\end{array}$ & $\begin{array}{l}62,3 \quad a \\
130,6\end{array}$ & $\begin{array}{l}59,4^{b} \\
84,7^{a}\end{array}$ & $\begin{array}{l}59,7 \\
74,7 b\end{array}$ & $\begin{array}{l}32,9^{a} \\
42,6^{a}\end{array}$ & $\begin{array}{l}39,2^{b} \\
57\end{array}$ & $\begin{array}{l}67^{a} \\
90,4^{a}\end{array}$ & $\begin{array}{r}7,8 * * * \\
13,9 * * *\end{array}$ \\
\hline
\end{tabular}

a Significativement différent du stade $(n-1)(\mathrm{P} \leqslant 0,01)$.

$b$ Siguificativement différent du stade $(n-2)(P \leqslant 0,01)$.

*** Effet global du stade ce prélèvement $(\mathrm{P} \leqslant 0,001)$.

$7^{\circ}$ La pression osmotique (fig. 6) de l'albumen diminue de $30 \mathrm{mOsm} / 1$ au cours du séjour de l'œuf dans l'utétus, et, principalement, entre 6 et I4 heures. Cette diminution peut être attribuée en partie à l'apport massif d'eau (renfermant cependant $\mathrm{Na}^{+}$et $\mathrm{K}^{+}$pendant la durée du "plumping ») et en partie aux diminutions de teneur en $\mathrm{Ca}^{++}$et $\mathrm{Cl}$ - qui interviennent à partir de $1 \mathrm{a} 6^{\mathrm{e}}$ et de la $8^{\mathrm{e}}$ heure respectivement.

$8^{\circ}$ Le pH de l'albumen (fig. 7 et tabl. I) reste inchangé et voisin $\mathrm{du} \mathrm{pH}$ sanguin $(7,40)$ pendant la phase de "plumping " $(6-I 2 \mathrm{~h})$. Il diminue ensuite jusqu'aux environs de la $20^{\mathrm{e}}$ heure pour atteindre une valeur minimale de $7, \mathbf{1} 2$ et reprend sa valeur 


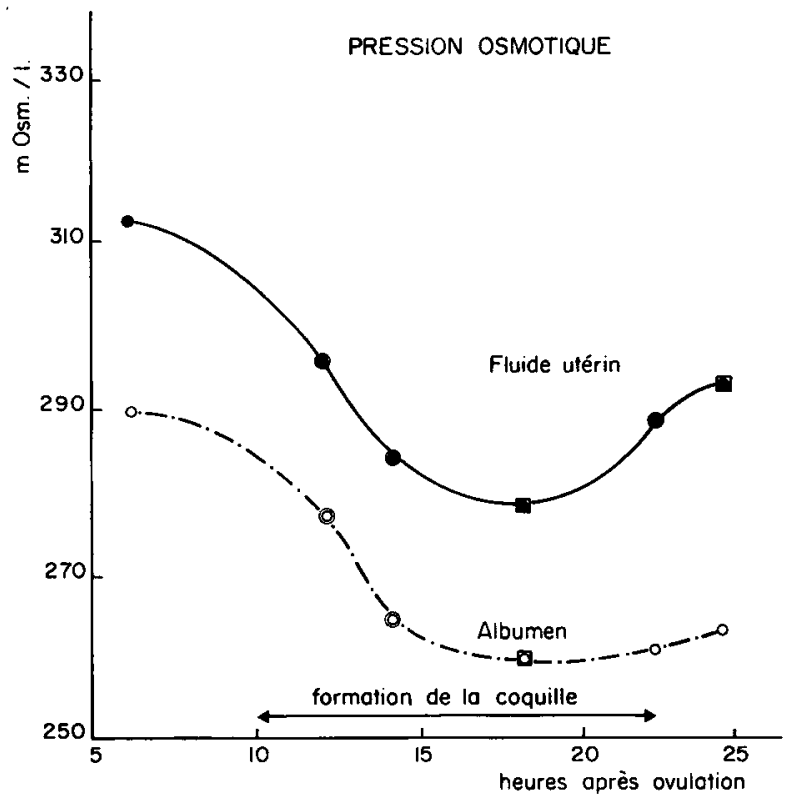

FIg. 6. - Pression osmotique de l'albumen et du fluide utérin (mOsm/l) en fonction du temps écoule depuis l'oviposition précédente

Légende : voir figure I

- - fluide utérin o-o albumen

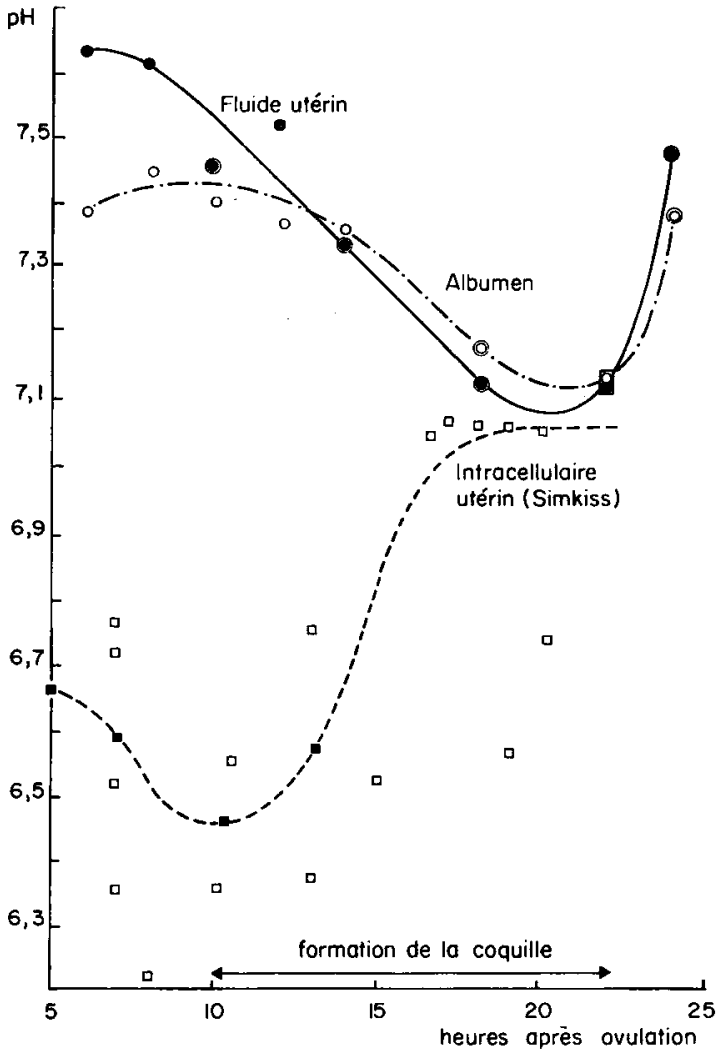

Fig. 7. $-\mathrm{pH}$ de l'albumen et du fuide utérin en fonction du temps écoulé depuis l'ovoposition précédente

fluide utérin o- $\mathrm{O}$ albumen - - $\mathrm{pH}$ intracellulaire d'après Simkıss (r969) 
initiale à 24 heures. Cet abaissement du pH de l'albumen entre $\mathrm{I} 2$ et 22 heures n'est accompagné d'aucune variation de $\mathrm{COO}_{2}$ (stable entre Ioo-I Io $\mathrm{mm} \mathrm{Hg}$ ) mais d'une réduction significative de la teneur du blanc en bicarbonate (tabl. I).

$9^{\circ}$ Les variations de composition du fluide utérin apparaissent particulièrement remarquables et de plus grande amplitude que celles de l'albumen de l'œuf. Elles suivent toutefois des directions identiques.

Nous constatons en particulier (fig. 7 et tabl. I) qu'une acidose intra-luminale se développe entre la Io et la $22^{\mathrm{e}}$ heure, caractérisée à $\mathrm{I} 8$ heures par un $\mathrm{pH}$ de 7 ,II et une teneur minimale en bicarbonate de 42,6 méq/ 1 sans modification significative de $p \mathrm{CO}_{2}$. Après 22 heures le $\mathrm{pH}$ et la teneur en $\mathrm{HCO}_{3}-$ du fluide utérin remontent brutalement aux valeurs du début de calcification. Ainsi les évolutions des $\mathrm{pH}$ de l'albumen et du fluide utérin sont exactement identiques.

La comparaison des figures 2 et 3 montre qu'entre 6 et I 8 heures le liquide utérin, comme l'albumen, s'enrichit considérablement en potassium et que, simultanément, sa teneur en sodium décroît $(+5 \mathrm{I}$ et - IO2 méq/1 respectivement). Cette dernière apparaît en permanence inférieure à celle de l'albumen (fig. 2) alors que la situation est inversée pour $\mathrm{K}^{+}$(fig. 3).

$\mathrm{Au}$ niveau de ce fluide utérin, la $\mathrm{I} 8^{\mathrm{e}}$ heure est également caractérisée par un pic de concentration du calcium qui succède à une première augmentation visible à Io heures (fig. 4).

Notons enfin que la pression osmotique de ce liquide est constamment supérieure de 20 à $25 \mathrm{mOsm} / 1$ à celle de l'albumen (fig. 6) et présente un minimum à la I8 heure, inférieur de $40 \mathrm{mOsm} / 1$ à la pression osmotique du plasma (320 $\mathrm{mOsm} / 1$ selon EL, JACK et LAKE, Ig66).

\section{DISCUSSION}

\section{I. - Équilibre acido-basique utérin}

Les valeurs de $\mathrm{PCO}_{2}$ du fluide utérin enregistrées ici semblent plus proches de celles calculées à partir des données de MUELLER (I967) (II3-II7 mm Hg) qu'à partir de calles de É, JACK et LAKE, I 967 (73 à $78 \mathrm{~mm} \mathrm{Hg}$ ). Compte tenu de l'imprécision des stades de prélèvaments choisis par ces auteurs, les valeurs de $\mathrm{pH}$ qu'ils rapportent sont, en revanche, toutes compatibles avec les nottres mais ne donnent aucune indication sur les fluctuations de $\mathrm{pH}$ liées au fonctionnement de l'utérus.

ses différents ions transférés des liquides extracellulaires à l'œuf transitent nécessairement par les liquides intracellulaires et le fluide utérin et la composition de ce dernier règle les conditions physico-chimiques de précipitation du carbonate de calcium de la coquille (Movgin, I968). Ce phénomène se traduit ici par une chute de concentration des ions $\mathrm{HCO}_{3}-$ du liquide utérin égale à $88 \mathrm{méq} / \mathrm{l}$, entre Io et I 8 heures après oviposition, c'est-à-dire pendant les 8 premières heures de formation de la coquille.

Il serait donc confirmé que des ions $\mathrm{HCO}_{3}-$ sont mobilisés in utero; on sait, par ailleurs, que ces ions proviennent vraisemblablement du $\mathrm{CO}_{2}$ métabolique (SImkIss, I96I ; HODGES et LörCHER, I967 ; LÖRChER, ZschEILE et BRONSCH, I970 ; KuTAS, 
KEMENY et LENCSÉs, I970). Cette observation est en accord avec 1'hypothèse de Diamanstein (I966), reprise par Mongrn (r969), suivant laquelle les cellules épithéliales utérines sécréteraient des ions $\mathrm{HCO}_{3}-$ provenant de l'hydratation intracellulaire du $\mathrm{CO}_{2}$ sous l'action de l'anhydrase carbonique. La baisse de $\mathrm{pH}$ intraluminal enregistrée entre to et 22 heures, est un argument supplémentaire puisqu'elle est conforme à la réaction :

$$
\mathrm{HCO}_{3}-+\mathrm{Ca}^{++} \rightarrow \mathrm{CaCO}_{3}+\mathrm{H}^{+}
$$

L'hypothèse de Srmkiss (1969, I970) qui suppose une sécrétion d'ions $\mathrm{OH}^{-}$vers la lumière utérine et leur combinaison directe avec le $\mathrm{CO}_{2}$ suivant la réaction.

$$
\mathrm{CO}_{2}+\mathrm{OH}^{-} \rightarrow \mathrm{HCO}_{3}^{-} \rightarrow \mathrm{CO}_{3}^{--}+\mathrm{H}^{+}
$$

peut également être soutenue par les résultats présents. Nous n'avons certes pas enregistré de variations significatives de la $p \mathrm{CO}_{\mathbf{2}}$ du fluide utérin au cours de la formation de la coquille mais la très grande dispersion des valeurs ne permet pas de conclure à la non-existence de telles variations.

Nous avons reporté sur la figure 6 les valeurs de $\mathrm{pH}$ intracellulaire utérin enregistrées par Simkiss : elles montrent que l'acidose intracellulaire est surtout importante pendant la durée du "plumping ". En fin de calcification (I8-22 h) les trois valeurs de $\mathrm{pH}$ de l'albumen, du fluide utérin et des liquides intracellulaires convergent vers une valeur unique voisine de 7 ,ro.

\section{II. - Importance des stades 18 et 10 heures}

On sait que la $22^{\mathrm{e}}$ heure qui suit l'ovulation est une étape caractéristique de la formation de l'œuf (Mongrn et LACASSAGNe, I966; HodGES I969, I970) et nous la retrouvons ici. Il apparait cependant que le stade $\mathrm{I} 8$ heures en est une autre, remarquable par les pics de $\mathrm{pH}$ que nous venons d'évoquer mais aussi par une teneur maxima du fluide utérin en $\mathrm{Ca}^{++}$et $\mathrm{K}^{+}$, et par un minimum de pression osmotique et de teneur en sodium de ce même milieu.

La $10^{\theta}$ heure qui marque le début de la croissance des cristaux de la coquille, est caractérisée par des mouvements importants de calcium. Il est possible qu'une faible part du calcium de l'albumen ait participé au préalable à l'initiation des nodules de calcification qui, selon Simriss (Ig68) intervient dans l'isthme ou à la jonction isthmo-utérine. Nous observons, quant à nous, une diminution du contenu calcique de l'albumen entre 6 et Io heures, donc lorsque l'œuf est déjà in utero, mais elle ne représente que l'équivalent de $4 \mathrm{mg} \mathrm{de} \mathrm{CaCO}_{3}$.

Il existe, par ailleurs, à la $_{\text {Io }}{ }^{\mathrm{e}}$ heure une brusque décharge de calcium dans le fluide utérin. Ėn fait la teneur de 8,4 méq $\mathrm{Ca}^{++} / 1$ observée à cet instant est la résultante de deux séries de valeurs dont les moyennes, égales à 4,5 et I2,4 méq/1, sont statistiquement différentes. Dans le premier cas, la croissance des cristaux doit déjà être commencée alors qu'elle ne l'est pas encore dans le second comme le prouvent les valeurs de $\mathrm{pH}$ et de teneur en $\mathrm{HCO}_{3}-$ du liquide utérin et le poids de la coquille.

\section{III. - Transferts ioniques vers l'albumen}

Les variations de concentrations électrolytiques observées dans l'albumen et le fluide utérin méritent quelques commentaires en fonction des résultats déjà connus. 
La composition ionique de l'albumen des œufs prélevés pendant le " plumping " ou en fin de calcification est dans l'ensemble en bon accord avec les résultats de DRAPER (I966), LEONARD (I968) et SAUVEUR (I970 b). L'analyse complète du séjour de l'œuf in utero effectuée ici permet d'avoir une vue plus précise des phénomènes.

Nos résultats montrent en particulier qu'entre I2 et 22 heures 1'albumen s'enrichit en potassium à raison de I4 $\mu$ éq/h/g de matière sèche alors que le sodium est réabsorbé suivant un flux exactement identique en valeur absolue. Il est probable cependant que le phénomène essentiel ne se situe pas au niveau des échanges albumenfluide utérin, compte tenu de la grande perméabilité des membranes coquillières, et même de la coquille, aux électrolytes (LEONARD, I968). Le fait même qu'entre I2 et 22 heures, le classement relatif de l'albumen et du fluide utérin en fonction de leur teneur en $\mathrm{K}^{+}$et $\mathrm{Na}^{+}$suive la direction des échanges, nous confirme dans l'idée que ces transferts doivent s'effectuer suivant de simples gradients chimiques.

Il n'en est sans doute pas de même au niveau de la paroi utérine. Nous assistons dans le fluide utérin lui-même à des variations antagonistes des teneurs en $\mathrm{Na}^{+}$et $\mathrm{K}^{+}$ et HodGES (I969, I970) a mis en évidence une mobilisation effective du potassium plasmatique à partir de $1 \mathrm{a}$ Io $^{\mathbf{e}}$ heure qui suit l'oviposition. D'après HuRwITZ, CoHEN et BAR (I970) il existe pendant la calcification de la coquille, une différence de potentiel électrique au niveau de la paroi utérine, compatible avec l'existence d'une " pompe " à sodium. Ces faits ne démontrent pas cependant qu'un transport couplé $\mathrm{Na}+/ \mathbf{K}+$ est effectué par la paroi utérine; la sécrétion très importante d'ion $\mathrm{Ca}^{++}$ et la réabsorption d'ions $\mathrm{H}^{+}$qui interviennent au même niveau, doivent être considérées dans l'ensemble des échanges.

Rappelons enfin que l'existence de la réabsorption de sodium que nous démontrons ici avait été supposée par Hoover et SMITH (I958), DRAPER (I966) et LEONARD (I968) mais que ces auteurs ne disposaient que de valeurs relatives au début et à la fin de la calcification.

Le transfert important d'eau effectué vers l'albumen entre 6 et I2 hetres ne peut pas être de nature simplement osmotique si l'on se fie aux deux courbes de la figure 6. Nous pensons avec LEONARD (I968) que, pendant cette période, l'eau doit être attirée à l'intérieur des membranes coquillières grâce aux capacités de gonflement du gel protéique que constitue l'albumen. Nous trouvons au début du "plumping " un $\mathrm{pH}$ maximum de 7,4 qui est le plus éloigné du point isoélectrique de protéines et par conséquent favorable à leur hydratation; la présence de $\mathrm{NaCl}$ peut favoriser ce processus en abaissant la répulsion électrostatique entre les groupements électronégatifs des protéines suivant le schéma décrit par HaMm (I963) pour le muscle.

L'intervention d'équilibres de DonNaN pourrait par ailleurs expliquer un appel d'eau et d'électrolytes diffusibles dans le compartiment contenant l'ion protéique non diffusible. A la suite de LEONARD (I968), nous envisageons comme possible cette intervention mais il resterait à la démontrer, de façon certaine.

Ce dernier auteur ne trouve pas de variation significative de pression osmotique de liquide utérin entre le début et la fin de calcification de la coquille. Les valeurs reportées à la figure 6 justifient ce résultat mais montrent qu'un écart existe cependant entre le début du " plumping " et le stade $\mathrm{I} 8$ heures. Ce résultat étant également valable pour l'albumen, nous pouvons en déduire que, globalement, le séjour de l'œuf in utero entraîne un abaissement de la pression osmotique de ce milieu, malgré 
l'apport très important de potassium et de glucose (DAvidson et DRAPER, I969) qui y est effectué.

Nous rappellerons pour terminer que tous ces phénomènes de transfert d'ions effectués in utero sont vraisemblablement dépendants de l'équilibre acido-basique de l'animal puisqu'une acidose métabolique entraîne une diminution de l'épaisseur de la coquille (HALL et HELBACKA, I959; HuNT et AITKEN, I962) et modifie la composition électrolytique de 1'albumen (SAUVEUR, I970 $a$ et $b$ ).

Ces altérations étant uniquement imputables aux transferts utérins (SAUVEUR, I970 b), d'autres recherches sont actuellement en cours afin d'étudier l'action de telles acidoses sur la composition du fluide utérin.

Reçu pour publication en février 1971.

\author{
SUMMARY \\ COMPARATIVE STUDY OF UTERINE FLUID AND EGG ALBUMEN \\ IN THE SHEI, GLAND OF THE HEN
}

The purpose of this work was to study water and ions transfers which occur in the uterus (shell gland) of the hen. Eggs at various stages of development and uterine fluid samples were drawn out of the shell gland, after laparatomy. Between 6 and 24 hours after the previous oviposition, six birds were used every two hours. To avoid doing samplings during the night, we used about 20 hens submitted to an inverted nycthemeral rhythm. On each sample of egg albumen and uterine fluid, we measured $\mathrm{pH}, p \mathrm{CO}_{2}$, osmotic pressure and the $\mathrm{HCO}_{8}^{-}, \mathrm{Na}^{+}, \mathrm{K}^{+}, \mathrm{Ca}^{++}$and $\mathrm{Cl}^{-}$ions levels.

Our results show that ionic fluxes take place in both the albumen and uterine fluid. They have opposite directions according to each considered stage; so $\mathrm{Na}^{+}$is secreted into the albumen between 6 and 12 hours, then reabsorbed between 12 and 22 hours after the previous oviposition. During this last period, an exchange $\mathrm{Na}^{+}-\mathrm{K}^{+}$(ratio $\mathrm{I} / \mathrm{I}$ ) occurs between albumen and uterine secretion; nevertheless it is not actually demonstrated that a coupled transport of $\mathrm{Na}^{+}$and $\mathrm{K}^{+}$exists in the shell gland epithelium.

The I 8 th hour is characterized by a minimum osmotic pressure and by a maximum acidosis due to a mobilization of $\mathrm{HCO}_{3}$ - ions. When eggshell growth begins (on the roth hour), we observe a decrease of the albumen calcium content and a sudden increase of the calcium level in the uterine fluid.

These results are discussed in relation to studies already made on the origin of $\mathrm{Ca}^{++}$and $\mathrm{CO}_{3}{ }^{-}$ions of the eggshell ; they confirm that $\mathrm{HCO}_{3}{ }^{-}$ions, originating from metabolic $\mathrm{CO}_{2}$, are probably secreted in the lumen by the uterine cells.

The nature of transfers which permit albumen hydratation in utero, is also discussed; it seems well established that, after the plumping period (6-1 $2 \mathrm{~h})$, cationic exchanges arise between egg albumen, uterine fluid and blood.

\title{
RÉFÉRENCES BIBLIOGRAPHIQUES
}

Beadle B. W., Conrad R. M., Scott H. M., 1938. Composition of the uterine secretion of the domestic fowl. Poultry, Sci., 17, 498-504.

Davidson M. F., Draper M. H., 1969. The accumulation of glucose in the white of the egg of the hen. J. Physiol., 202, II9-I20 p.

Diamanstein T., 1966. Utber die lokale rolle der carboanhydratase im hinblick auf die eischalenverkalkung. Arch. Geflugelk., 30, 309-320. 
Draper M. H., I966. The accumulation of water and electrolytes in the egg of the hen. In Physiology of the domestic forol (B. E. M. B. Symposium I), 63-74; Oliver and Boyd, Edinburgh and London.

EL JACK M. H., LAKE P. E., I966. The distribution of the principal inorganic ions in venous blood of the adult domestic cock and the content of carbon dioxide in the plasma. Brit. Poult. Sci., 7, 315-318.

EL JACK M. H., LAKE P. E., I967. The content of the principal inorganic ions and carbon dioxide in uterine fluids of the domestic hen. J. Reprod. Fert., 13, I27-132.

Hall K. N., Helbacka N. V., I959. Improving albumen quality. Poultry. Sci., 38, III-II4.

Hamm R., I963. The water imbibing power of foods. In Leitch J. M., Rhodes D. N. Recent Advances in Food Sciences, vol. 3, 218-229, Butterworths, London.

Hodges R. D., 1969 . $\mathrm{pH}$ and mineral ion levels in the blood of the laying hen (Gallus domesticus) in relation to egg shell formation. Comp. Biochem. Physiol., 28, 1243-1257.

Hodges R. D., I97o. Blood pH and cation levels in relation to egg shell formation. In Symposium on the physiology of the egg shell formation. Ann. Biol. anim. Biochim. Biophys., 10, $\mathrm{n}^{\circ}$ hors-série 2 , I99-2I3.

Hodges R. D., Lörcher K., I967. Possible sources of the carbonate fraction of the egg shell calcium carbonate. Nature, 216, 609-6ro.

Hoover G. N., Smith A. H., 1958. Secretion of fluid by the shell gland of the laying hen. Poultry Sci., 37, 467-47I.

Hunt J. R., Aitken J. R, r 962 . The effect of ammonium and chloride ions in the diet of hens on egg shell quality. Poultry, Sci., 41, 434-438.

Hurwitz S., Conen I., BaR A., I970. The transmembrane electrical potentiel difference in the uterus (shell gland) of birds. Comp. Biochem. Physiol., 35, 873-878.

Kutas F., Kemény A., Lencstes G. Y., I97o. Studies on the influence of egg shell formation on the acid-base balance of the laying hen. Acta Vet. Acad. Scient. Hung., 20, 28I-287.

LEONARD Elizabeth M., I968. The accumulation of minerals in avian oviduct. Ph. D. Thesis, Edinburgh.

LÖrcher K., Zscheile C., Bronsch K., I97o. Transfert of continuously i. v. infused $\mathrm{NaHC}^{14} \mathrm{O}_{3}$ and $\mathrm{Ca}^{47} \mathrm{Cl}_{2}$ to the hen egg shell. In Symposium on the Physiology of the egg shell formation. Ann. Biol.anim. Biochim. Biophys., 10, $\mathrm{n}^{\circ}$ hors série 2, $193-198$.

Mongin P., r 968 . Role of acid-base balance in the physiology of egg shell formation. World's Poultry. Sci. J., 24, 200-230.

Mongin P., I969. Physiologie de la formation de la coquille chez les oiseaux. Rôle de l'équilibre acidobasique. Thèse de Doctorat ès-Sciences, Paris.

Mongin P., Lacassagne L., r966. Équilibre acido-basique du sang et formation de la coquille de l'œuf. Ann. Biol. anim. Bioch. Biophys., 6, 93-100.

Mongin P., Sauveur B., I97o. Composition du fluide utérin et de l'albumen durant le séjour de l'œuf dans l'utérus chez la poule domestique. C. R. Acad. Sci., Paris, Sér. D., 270, 17×5-xy 8.

Muelder W. J., r967. Effects of spironolactone on laying pullets. Poultry. Sci., 46, 742-749.

SAUveur B., r969. Étude de la composition électrolytique des différentes zones de l'albumen de l'œuf chez deux races de poules. Ann. Biol. anim. Bioch. Biophys., 8, 563-573.

Sauveur B., I97o $a$. Acidoses métaboliques expérimentales chez la Poule pondeuse. II. Action sur la composition minérale de l'albumen de l'œuf. Ann. Biol. anim. Bioch. Biophys., 10, 81-1oo.

Sauveur B., t97o $b$. Action d'une acidose métabolique sur les transferts d'ions effectués dans l'oviducte de la Poule. In Symposium on the Physiology of the egg shell formation. Ann. Biol. anim. Bioch. Biophys., 10, $\mathrm{n}^{\circ}$ hors-série 2, $215-227$.

Simkiss K., rg6r. Calcium metabolism and avian reproduction. Biol. Rev., 36, 32r-367.

Simkiss K., Ig68. The structure and formation of the shell and shell membranes. In CARTER T. C., Egg quality : a study of the hen's egg, 3-25, Oliver and Boyd, Edinburgh.

Simkiss K., r969. Intracellular pH during calcification. A study of the avian shell gland. Biochem: J., $111,647-652$.

Simkiss K., I97o. Effect of acetazolamide on intracellular pH of avian shell gland. J. Physiol., 207, 63-64 P. 